\title{
The role of structural context in perception: Syntax in the recognition of algebraic expressions
}

\author{
MICHAEL RANNEY \\ University of Pittsburgh, Pittsburgh, Pennsylvania
}

\begin{abstract}
Two character-identification experiments investigated the function of structural context during the processing of briefly exposed algebraic strings. Neither experiment provided evidence to support the notion of an algebra-superiority effect, a contextually driven enhancement of the recognition of specific algebraic characters. However, the results of Experiment 2 indicate that the structure of algebra does provide information at the level of a character's categorical denomination. These findings suggest that the parsing of an algebraic string includes a level of processing in which its structural context places restrictions on the denominations of its symbols. A processing model of algebraic perception is proposed that incorporates these syntactic constraintsconstraints that appear to be independent of feature-based character identification processes.
\end{abstract}

With respect to perceptual information processing, two kinds of contextual effects can be distinguished. Effects of the first kind are mediated by one's knowledge of rather specific patterns. An effect of this sort may be found in the perception of a letter in the context of a correctly spelled word. Many psychologists (see reviews by Estes, 1975; McClelland \& Rumelhart, 1981; Paap, Newsome, McDonald, \& Schvaneveldt, 1982) have demonstrated that the recognition of letters in a word is superior to the recognition of letters embedded in a nonword. This particular fixed-pattern (i.e., lexical) contextual phenomenon is often called the word-superiority effect. The second kind of contextual effect, the one that this article primarily addresses, involves a more generalized form of context. This sort of structural context appears to play a crucial role in the recognition of words that are embedded in a syntactically correct sentence. Miller and Isard (1963), for instance, found that spoken words are better detected in grammatical sentences than in ungrammatical strings. Examples such as these illustrate that both fixed and structural patterns are utilized during the comprehension of natural language.

Although psychologists have observed the perceptual effects of fixed patterns for over a century (Cattell,1885),

\footnotetext{
Some of the data described in this article were presented by the author and James Greeno at the May 1984 meting of the Midwest Psychological Association in Chicago, Illinois. The research was supported by the Learning Research and Development Center, and by Grant NR667-534 from the Office of Naval Research, for which the author is most grateful.

Many thanks are offered to J. Greeno, who provided insightful guidance throughout the term of this research, as well as helpful comments and advice on numerous versions of this article. Additional thanks are offered to S. Chaiklin, D. Klopfer, A. Lesgold, M. Million, R. Mumaw, C. Perfetti, several anonymous reviewers, and members of the LRDC Informal Seminar, whose suggestions and criticisms of early versions also enhanced the present article. Requests for reprints should be sent to Michael Ranney, 808 LRDC, 3939 O'Hara Street, Pittsburgh, PA 15260.
}

the study of the perceptual nature of the structural context of (written) English remains problematic. Most of the perceptual work on graphemic strings has involved tachistoscopic presentations, requiring relatively compact stimuli. English sentences appear to be too long for such purposes; even relatively short sentences tend to encompass dozens of characters, and so require several eye fixations and saccades. In order to better study the structural aspects of contextually driven perception, a more compact (or less redundant) language would be preferred. The domain of algebra provides just such a language.

Algebraic expressions are graphemic strings of symbolic characters that are governed by a grammar. One can determine whether or not an expression is algebraically grammatical, just as one can tell if a noun phrase is grammatical. The syntax of algebra places certain conventional ordering constraints on its elements, much as English syntax does. (See Table 1 for some examples of grammatical and ungrammatical algebraic strings.)

It is algebra's compactness, however, that makes it particularly appropriate for the study of the perceptual effects of structural context. Even rather complex expressions can be formed with the appropriate concatenation of relatively few characters. In algebra, then, we have a formal, syntactic language that is compact enough to be briefly perceived (i.e., with fewer eye fixations and saccades than are required to perceive English sentences). These linguistic and visual characteristics have motivated the present use of algebraic strings as perceptual stimuli.

Table 1

Examples of Algebraic and Nonalgebraic Stimuli

\begin{tabular}{cc}
\hline Algebraic & Nonalgebraic \\
\hline $5 L+(9) K$ & K9)L(5+ \\
$3(X Y+6)$ & Y3) $-X 6($ \\
$2-8(J K)$ & $(J 8 K 2+)$ \\
V+(4) $7 W$ & WV7) $(4-$ \\
$(3 M-4 N)$ & N3M +$) 4($ \\
\hline
\end{tabular}


Data collected by Bernard (1983) show that grammatical algebraic strings can facilitate information processing at some level. These data indicate that an expression's algebraic structure yields recall superior to the recall of nonexpressions. This sort of recall enhancement has been observed in a number of domains, such as chess, Go, and circuit diagrams, when materials of varying "meaningfulness" have been contrasted (Chase \& Simon, 1973; Egan \& Schwartz, 1979; Reitman, 1976). Subjects in Bernard's experiment viewed either equations or nonalgebraic strings (from 4 to 15 characters in length) for $1.5 \mathrm{sec}$, and were then asked to report them. The algebraic expressions were recalled much more accurately than were their nonsensical counterparts. Of course, this finding might well be an effect of memorial elaboration, rather than one of perception. The enhanced recall of algebra is quite likely a manifestation of better chunking strategies and/or a more elaborate representation for such stimuli. Given the relatively long exposure duration, it is not clear that the subjects perceived the algebraic stimuli any beiter than they perceived the nonalgebraic stimuli.

In contrast to Bernard's (1983) experiment, in which a great deal of postperceptual processing seems to have been invoked, the present work specifically attends to the earlier, more perceptual phases of algebraic parsing. This is accomplished by focusing on the recognition of an expression's characters.

A reasonably useful analogy can be drawn between the recognition of algebraic strings and the recognition of English words. To this end, Reicher's (1969) wordsuperiority phenomenon is particularly helpful, as is McClelland and Rumelhart's (1981) account of it. According to McClelland and Rumelhart's hierarchical interactive activation model, the word-superiority phenomenon is largely due to greater top-down activation received by letter nodes from known words, compared to their nonword counterparts. Remarkably, this model includes no explicit form of orthographic knowledge; it is an individual's vocabulary that actually drives the observed contextual advantage.

Within the domain of algebra, however, one would not expect even an expert subject to have a fixed lexicon, a knowledge structure that included all of the specific patterns that the individual could recognize. The class of correctly spelled words is but a finite set of the orthographically acceptable possibilities, whereas there are an infinite variety of algebraically acceptable expressions. It seems unlikely that an expression such as $7 x+5 y$ would be represented as a preexisting pattern in the same way that the word take might be.

A more plausible model for the individual's representation of algebraic expressions employs the notion of a generic lexicon, a collection of schemata that map onto algebraic strings of specified lengths. Each schema would have a set of serially positioned slots that could be filled only by characters of the appropriate category. For instance, instead of utilizing a specific lexical entry, such as $9 y z+4$, one might access the five-character schema numeral-letter-letter-operator-numeral. Thus, algebra users would have a vocabulary of expression forms, rather than one of specific expressions. Embedded within an algebraic variant of the interactive activation model, these forms would be capable of sending top-down contextual facilitation to individual character nodes on the basis of their categorical denomination, as illustrated in Figure 1.

Yet another account of algebraic recognition, the gener-

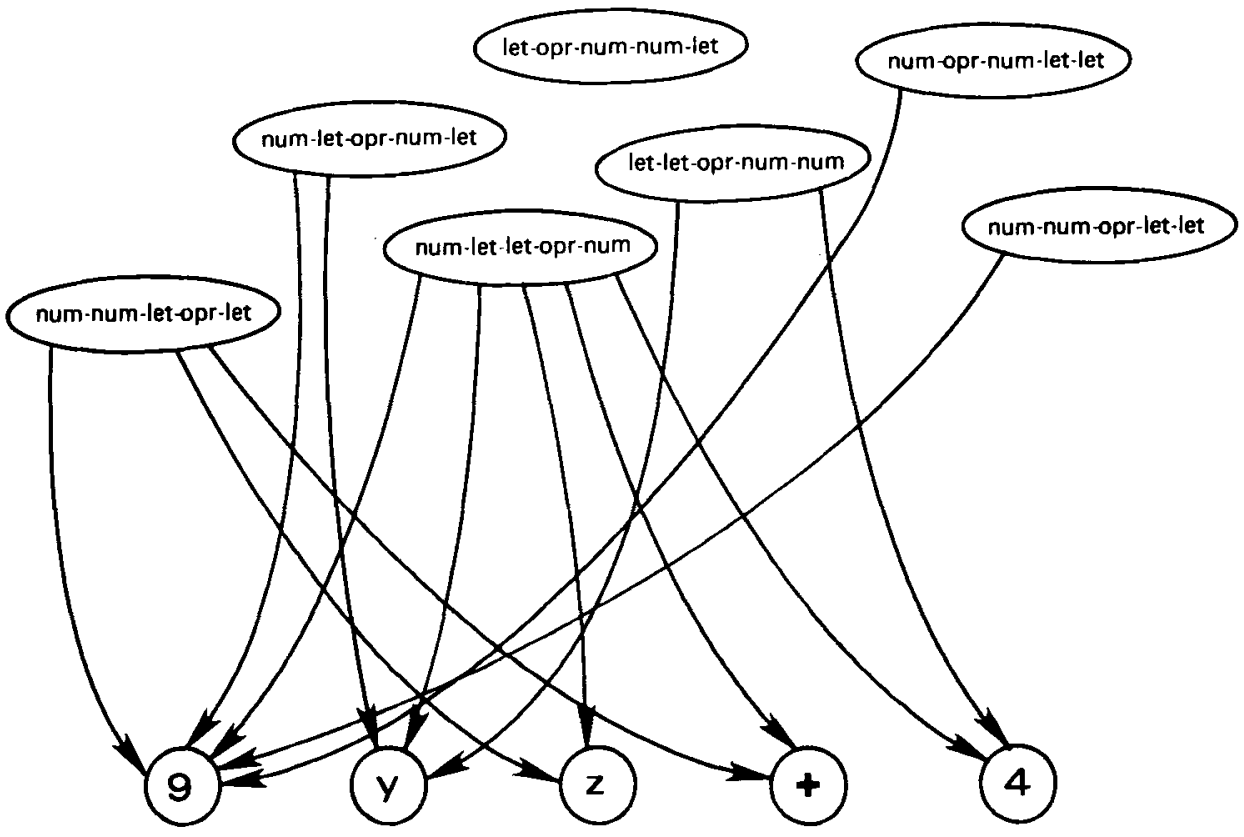

Figure 1. Pathways of top-down activation from various hypothetical generic lexical entries to elements of the algebraic expression $9 y z+4$. 
ative parse model, completely does away with the notion of a static lexicon. The model highlights the major difference between parsing a string of characters and recognizing a fixed pattern; an algebraic expression, unlike an English word, is a formal, generative entity whose meaning is reflected in its elements and their intercharacter relationships.

The generative parse model suggests that an expression's representation is created de novo, generated by some (possibly binary) parsing mechanism. The resulting structure would be a parse tree whose visual primitives and intermediate concepts are combined in such a way that the superordinate node, a node corresponding to what one might ordinarily think of as a lexical entry, encompasses the entire expression. In fact, it is conceivable that this superordinate node, once generated, might provide a form of contextual facilitation to its subordinate character nodes.

Each of these three models of algebraic recognition (fixed lexicon, generic lexicon, and generative parse) is capable of accounting for the contextually enhanced perception of characters in algebra. However, the only empirical data that support the existence of this facilitationthe recall data provided by Bernard (1983)-are confounded with short-term memorial processes. In the first of two Reicher-like experiments, I hoped to obtain an unconfounded measure of the hypothesized enhancement effect. Instead of words and nonwords, seven-character algebraic and (quasi-scrambled) nonalgebraic strings were employed as stimuli. In essence, the procedure was designed to determine whether or not an algebrasuperiority effect exists. If such an effect were obtained, characters in algebraic expressions would be better recognized than would characters in strings that violated the intercharacter syntax of algebra. The forced-choice decision in this experiment was always between target and foil letters (variables) or target and foil numerals (coefficients); the foil alternative, if inserted into the target's position, would yield a string that would be relatively equivalent, in its meaningfulness, to the tachistoscopically exposed stimulus.

\section{EXPERIMENT 1}

\section{Method}

Subjects. Sixteen students from an introductory psychology course volunteered to participate in this experiment, satisfying a portion of their class requirements. Each subject was familiar with the domain of algebra and was concurrently enrolled in a mathematics course. Typical subjects had completed more than two semesters of calculus.

Materials and Apparatus. Two types of seven-character stimuli, algebraic and nonalgebraic, were tachistoscopically presented on a TERAK 8510/a CRT. The algebraic stimuli were regular in that they violated no intercharacter ordering constraints (i.e., they were algebraically grammatical). Nonalgebraic strings were permutations of well-formed algebraic strings and violated a criterion of three algebraic ordering constraints.
Each stimulus was composed of two letters, two numerals, a pair of parentheses, and either a plus or a minus sign. ${ }^{1}$ The letters for a given stimulus were alphabetically (although not necessarily positionally) adjacent and were semirandomly chosen from either $\{J, K, L, M, N\}$ or $\{V, W, X, Y, Z\}$. The numerals for a stimulus (which never formed a two-digit number) were randomly chosen from the set $\{2,3,4,5,6,7,8,9\}$. Algebraic and nonalgebraic stimuli were counterbalanced with respect to the specific characters they included.

The following intercharacter constraints were met by all algebraic stimuli: (1) A letter could never directly precede a numeral, (2) an operator had to have its appropriate number of arguments, and (3) an opening parenthesis could never precede a closing parenthesis. A few grammatically sound but oddly constructed algebraic strings (such as $+2 \mathrm{x}(\mathrm{y}) 6)$ were deleted from the list of stimuli. Thirtyfive algebraic "character frames" (categorical templates) were eventually selected, as were 105 nonalgebraic frames. Some examples of the stimuli are provided in Table 1.

Design. Three experimental variables were factorially $(2 \times 2 \times 7)$ combined: stimulus type (algebraic or nonalgebraic), target category (numeral or letter), and target position. The experiment included 196 experimental trials, with each cell represented by seven (binary) accuracy values, this study's sole dependent measure.

Procedure. Prior to starting the experiment, subjects were given descriptions of the nature and composition of the trials and the stimuli to be presented. The experiment consisted of two blocks of trials, separated by a 5-min rest period. The first block began with 56 practice trials, followed by 84 experimental trials; the second block began with 7 practice trials, followed by 112 experimental trials.

Stimulus exposures were manipulated so that a subject's responding was correct on approximately $75 \%$ of the trials. Exposures were surreptitiously adjusted every $N$ trials, according to the formula

$$
d_{1}=d_{0}[1+.75(.75-n / N)]
$$

(from Rumelhart \& McClelland, 1982), where $d_{1}$ is the new duration, $d_{0}$ is the old duration, and $n$ is the number of correct responses from the previous set. Experimental replications were of 28 trials each $(N=28)$, but stimulus durations were adjusted every 7 trials $(N=7)$ during practice.

The task was self-paced, with subjects triggering each trial by striking a designated key on the terminal's keyboard. (Table 2's same condition illustrates the temporal sequence of a typical trial's events.) At the start of a new trial, two fixation-field delimiters immediately disappeared, and the stimulus appeared $175 \mathrm{msec}$ later. After an exposure of the appropriate length, the stimulus was masked by seven octothorpes (\#s). Two forced-choice alternatives followed the patterned mask by $500 \mathrm{msec}$, appearing above and below a masked character's horizontal position. The alternatives were either both letters or both numerals, and the subject was required to respond by typing the character that had appeared in the masked position. After the subject chose an alternative, the CRT screen returned to the starting configuration. Subjects were given no performance feedback.

Only those positions that contained either a letter or a numeral were probed, and the targeted character was always an alternative. An incorrect alternative, or foil, never corresponded to a character that was employed in the stimulus, yet was constrained to be an element of the character sets delineated above. Letter foils were alphabetically adjacent to letters in the stimulus, whereas numeral foils were randomly generated. Finally, alternative characters were counterbalanced across algebraic/nonalgebraic stimulus types.

A retrospective interview followed each participant's experimental session. The subjects were asked to verbalize their performance strategies (e.g., “Which characters did you try to focus on?') as 
well as any judgments about the relative difficulty of the probe conditions.

\section{Results and Discussion}

The experiment's most interesting result was that characters embedded in algebraic expressions were not better recognized than those in nonalgebraic strings $\mathbf{7 4 . 0 \%}$ vs. 73.7\%; $\left.C l_{.95}=\left[-0.024<\mu_{\mathrm{A}}-\mu_{\mathrm{NA}}<0.031\right]\right)$. In other words, no evidence was found to support the existence of an algebra-superiority effect. This might seem rather surprising, given that each of the models described above was, at the very least, consistent with some sort of contextually enhanced algebraic character recognition. Both of the lexical accounts, fixed and generic, assumed that an expression's algebraic representation might provide some top-down facilitation to the character level. Similarly, if a representation-building, generative sort of parsing were going on during these brief exposures, one might expect that the generated entities would provide some contextual enhancement by the time of the forced choice.

Thus, the absence of the hypothesized algebrasuperiority effect may well be attributed to the lack of any top-down facilitation. Of course, the absence may also be attributable to particular characteristics of the chosen methodology. Perhaps the proposed contextual enhancement processes actually exist, but are activated too slowly, due to the temporal characteristics of the task, ${ }^{2}$ to be of benefit. If so, it would appear that the kind of representation that allows subjects to recall algebraic expressions more accurately than nonalgebraic strings is either not generated or not accessible within this recognition experiment's temporal framework. The data are clear in one respect, however: Users of algebra do not employ algebraic lexicons in the same way (or not, at least, with the same speed) that they employ their English vocabularies.

Although the contextual manipulation yielded no main effect, it appeared as a potentially important interactive factor. A repeated measures analysis of variance showed that, although the stimulus type $\times$ target category interaction was marginal $[F(1,15)=4.50, M S e=.918, p=$ .051 ; see Figure 2], a very significant stimulus type $\times$ target category $\times$ serial position interaction was obtained $[F(6,90)=3.85, M S e=1.104, p<.0025]$. These interactions may be viewed primarily as subtle artifacts of the experiment's (algebraic and nonalgebraic) stimuli, rather than as the results of some theoretically interesting contextual effect. To be specific, if an algebraic term contained a numeral, it was never directly preceded by a letter; yet this was often the case for numerals in nonalgebraic (term-like) strings of numerals and letters. Given this structural difference between the stimulus types, if the usual serial position effect were observed, one would expect an enhanced recognition of algebraic coefficients (partially at the expense of variable recognition) relative to the recognition of nonalgebraic numerals and letters. (Overall, the recognition of numerals was superior to the recognition of letters $[78.4 \%$ vs. $69.2 \%$; $F(1,15)=31.24, M S e=1.502, p<.0001]$.) Since a highly significant and typical serial position effect was indeed observed $[F(6,90)=8.02, M S e=2.114$,

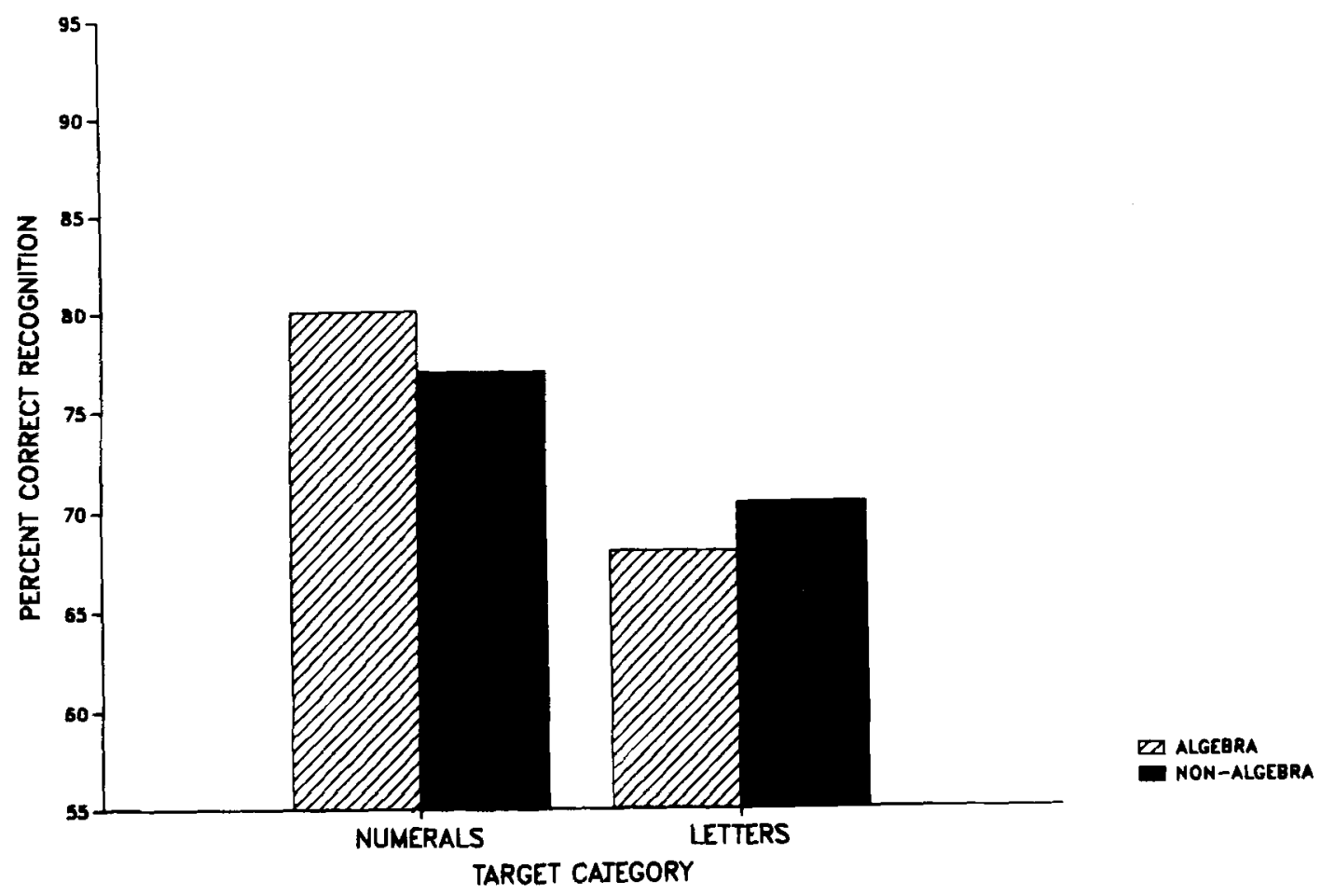

Figure 2. The percentage of correct character recognition as a function of stimulus type and target category. 
$p<.0001]$, this appears to be a reasonable explanation of the stimulus type $x$ target category interaction shown in Figure 2.

\section{EXPERIMENT 2}

The results of Experiment 1 suggest that an algebraic expression's context has no effect upon its perception. Yet these results do not completely rule out such top-down perceptual effects. In Experiment 1, which followed the Reicher paradigm as closely as possible, the two alternatives in a trial were of the same category-either letters or numerals. This ensured that either the foil or the target, inserted into the target position in the stimulus, would form a viable string. Thus, an observed contextual effect could be directly ascribed to the top-down facilitation of a character's recognition via some representation of its expression. Experiment 2 was designed, in part, to allow us to consider the notion of a contextual function that is relatively independent of such recognition - one that does not facilitate the identification of specific characters.

A relatively noninteractive contextual process might only provide the parser with a few of an algebraic expression's global features. These features might be useful in comprehending the expression's structure, but not its individual characters. Would Experiment 1's design have been sensitive to a facilitation based upon such features? No, because in the absence of a semantic context (e.g., that of an algebraic story problem), the only rules governing the form of an expression are syntactic in nature. These conventions govern the relative and ordinal positions of an expression's elements, but only with respect to their categorical denominations, not their precise identities. Consider the expressions $9 y z+4$ and $7 \mathrm{yz}+4$. In the absence of an embodying situation, both of the expressions are syntactically acceptable. With respect to the design of Experiment 1, a purely syntactic form of contextual facilitation would not have discriminated between the two. That is, knowing that a numeral appeared in the first position of an expression would hardly help a subject determine whether the numeral was a 9 or a 7. This realization prompts a narrower question: Does the syntactic structure of algebra constrain the parsing of algebraic expressions?

The string $9 y 3+4$ is not a syntactically acceptable entity in the world of algebraic expressions. Unlike a wellformed string, such as $9 y z+4$, it violates the algebraic convention that prohibits coefficients from following variables. Were a syntactically sensitive contextual process available, it would probably enhance the perception of $z$ in the latter string, relative to the perception of 3 in the former. This hypothesis is readily testable. The experimental question may be phrased, Does the structure of an algebraic expression enhance the recognition of its characters' categorical denominations? The paradigm used in Experiment 1 was modified to permit an answer to this question.
In order to allow a subject to respond on the basis of a character's category, a forced-choice trial must employ alternatives that differ in their categorical denomination. Therefore, two novel types of trials were introduced in Experiment 2, both of which involved intercategorical discrimination. Different trials provided as alternatives the targeted character and a foil that belonged to another category (i.e., a numeral vs. a letter). Categorical trials merely required the subject to respond with the targeted character's categorical denomination. These trial types allow us to address questions regarding interactions among a parser's sources of information. That is, data from the categorical trials can determine whether an effect of algebraic context across the different trials should be ascribed to an independent syntactic process or to one that depends upon explicit cues (i.e., particular forced-choice alternatives).

In addition to these types of trials, the present experiment also included the same-category trials that were employed in Experiment 1. This inclusion allowed a replication of the lack of an algebra-superiority effect with different subjects and in the context of varying types of trials. A replication of the previous finding is crucial to the above argument for a relatively independent contextual process.

Table 2 illustrates the three kinds of trials that were used. Based on the assumption of an autonomous, syntactically sensitive form of facilitation, one would expect (1) a replication of the lack of effect for the same condition's contextual manipulation, and (2) a significant perceptual enhancement for algebraic stimuli whose characters were probed in the different and categorical conditions (i.e., an enhancement relative to the perception of nonalgebraic stimuli in those conditions). However, if the latter intercategorical effects also proved insignificant, one could conclude that even a syntactic advantage does not enhance the perception of an algebraic expression's characters. Then again, if all three conditions yielded an effect for context, and a replication of the previous study were not obtained, the case for either lexically or generatively driven recognition might be resurrected.

It should be noted that Experiment 1's negative finding can be interpreted only with respect to the implicit assumption that the experimental materials and procedures were comparable to those utilized in word-superiority experiments. It is conceivable, though, that the results ob-

Table 2

\begin{tabular}{|c|c|c|c|}
\hline & Same & Different & Categorical \\
\hline Fixation field & : & $:$ & : \\
\hline Stimulus & $3(X Y+7)$ & $3(X Y+7)$ & $3(X Y+7)$ \\
\hline Mask & \#\#\#\#\#\#\# & 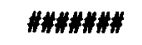 & \#\#\#\#\#\# \\
\hline Alternatives & 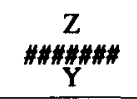 & 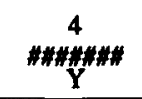 & 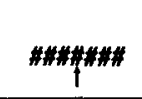 \\
\hline
\end{tabular}


tained in Experiment 1 were due to ill-chosen visual and/or procedural parameters. For instance, it may be that seven-character stimuli are just too complex to yield an effect of context, even for words and nonwords. To test this possibility, I carried out a seven-letter wordsuperiority experiment, which I will only briefly describe. ${ }^{3}$ It was essentially a replication of Reicher's (1969, no-cue condition) experiment, thus employing a procedure comparable to both that of Experiment 1 and that of the present experiment's same condition. In fact, the subjects and apparatus employed in the wordsuperiority experiment also served in Experiment 2.

The experiment yielded what appears to be the largest word-superiority effect ever reported (18\%; see Johnston, 1978). Letters embedded in words were correctly recognized $85.9 \%$ of the time, whereas the nonword letter recognition rate was only $67.9 \%$. The results bear out the adequacy of the basic procedural parameters employed in Experiments 1 and 2, so that one would be hard pressed to attribute the negative findings across same trials to deficient methodology. ${ }^{4}$

\section{Method}

Aside from the changes specified, the methodology of Experiment 2 was identical to that of Experiment 1.

Subjects. Fourteen paid volunteers from a (rather homogeneous) second-semester undergraduate honors calculus course served as subjects in this experiment.

Materials and Apparatus. A chinrest was added to the apparatus and positioned so that, for each subject, a given stimulus subtended a visual angle of approximately $2^{\circ}$.
Design. The experiment's design factorially $(2 \times 2 \times 3 \times 7)$ combined the following factors: (1) stimulus type (algebraic or nonalgebraic), (2) target category (numeral or letter), (3) trial type (same, different, or categorical), and (4) target position. Three replications of 84 experimental trials each contained three (mixed) sets of 28 via a Greco-Latin square.

Procedure. In total, 308 trials were segmented into three blocks by two 5 -min breaks. Each block began with practice trials during which the exposure duration was updated after every seven presentations. The first block began with 42 practice trials and the last two blocks began with 7 each. Eighty-four experimental trials followed each of the practice sets, with exposure durations being adjusted after each set of 28 .

For both same and different trials, in which two alternative characters appeared above and below a masked character's particular horizontal position (see Table 2), the subject was asked to type the character seen. During categorical trials, in which an arrow was placed directly beneath a particular (masked) character, the subject was asked to decide whether the indicated position held a numeral or a letter, and to respond appropriately by typing one of two labeled keys. Following a response, the subject was asked to confirm his or her choice, and the next trial began.

\section{Results}

Primary statistics. Of foremost interest is the manner in which specific levels of stimulus type and trial type interacted, as illustrated by Figure 3. [Not surprisingly, given the task's particular and synergistic characteristics, the full $2 \times 3$ interaction was not quite statistically significant: $F(2,26)=2.99, M S e=.322, p=.068$ ]. As in the previous experiment, ${ }^{6}$ across the intracategorically tested same trials, algebraic characters were recognized no more accurately than were characters from nonalgebraic strings

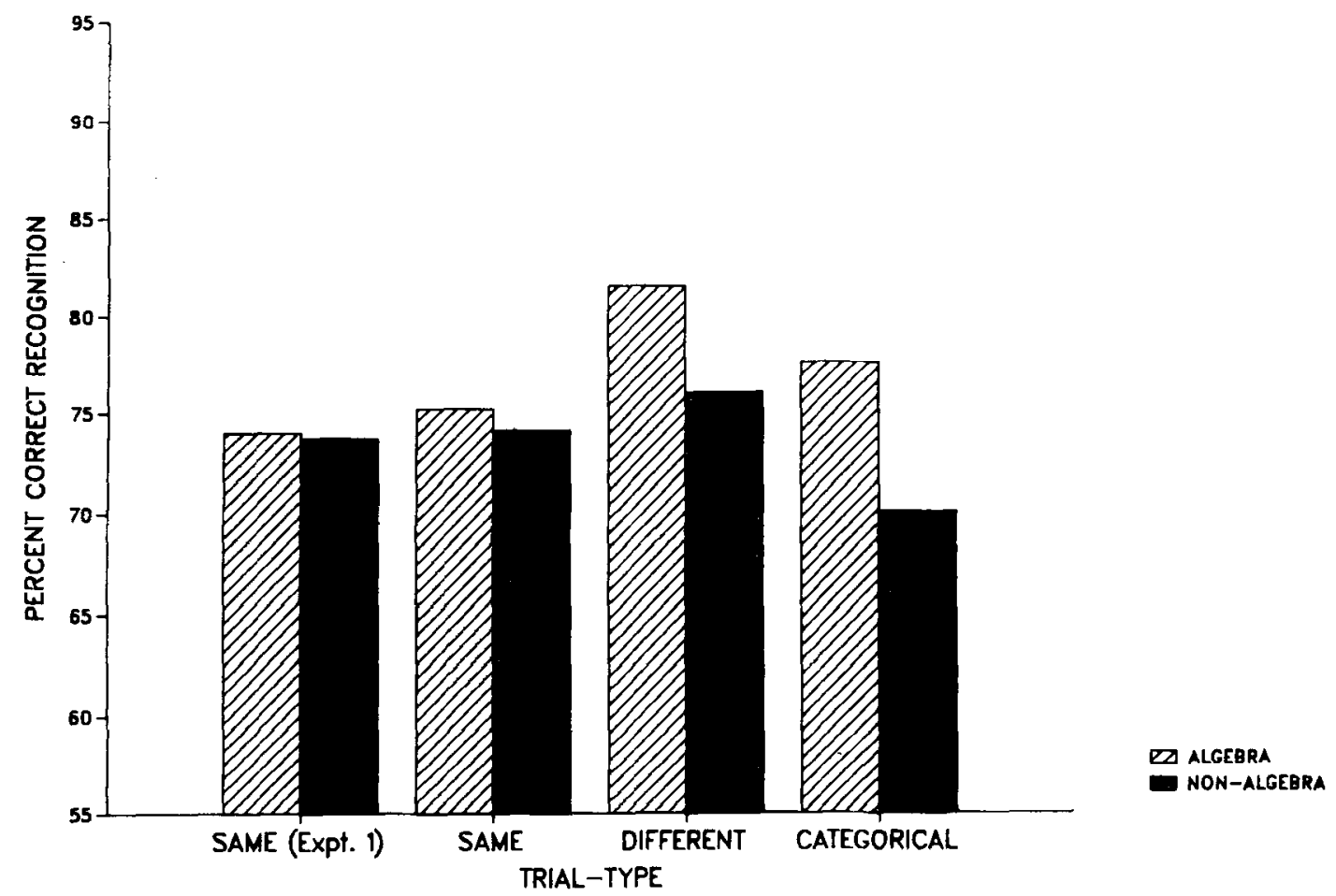

Figure 3. The percentage of correct recognition as a function of stimulus type and trial type. (Data from Experiment 1 are included for comparison.) 


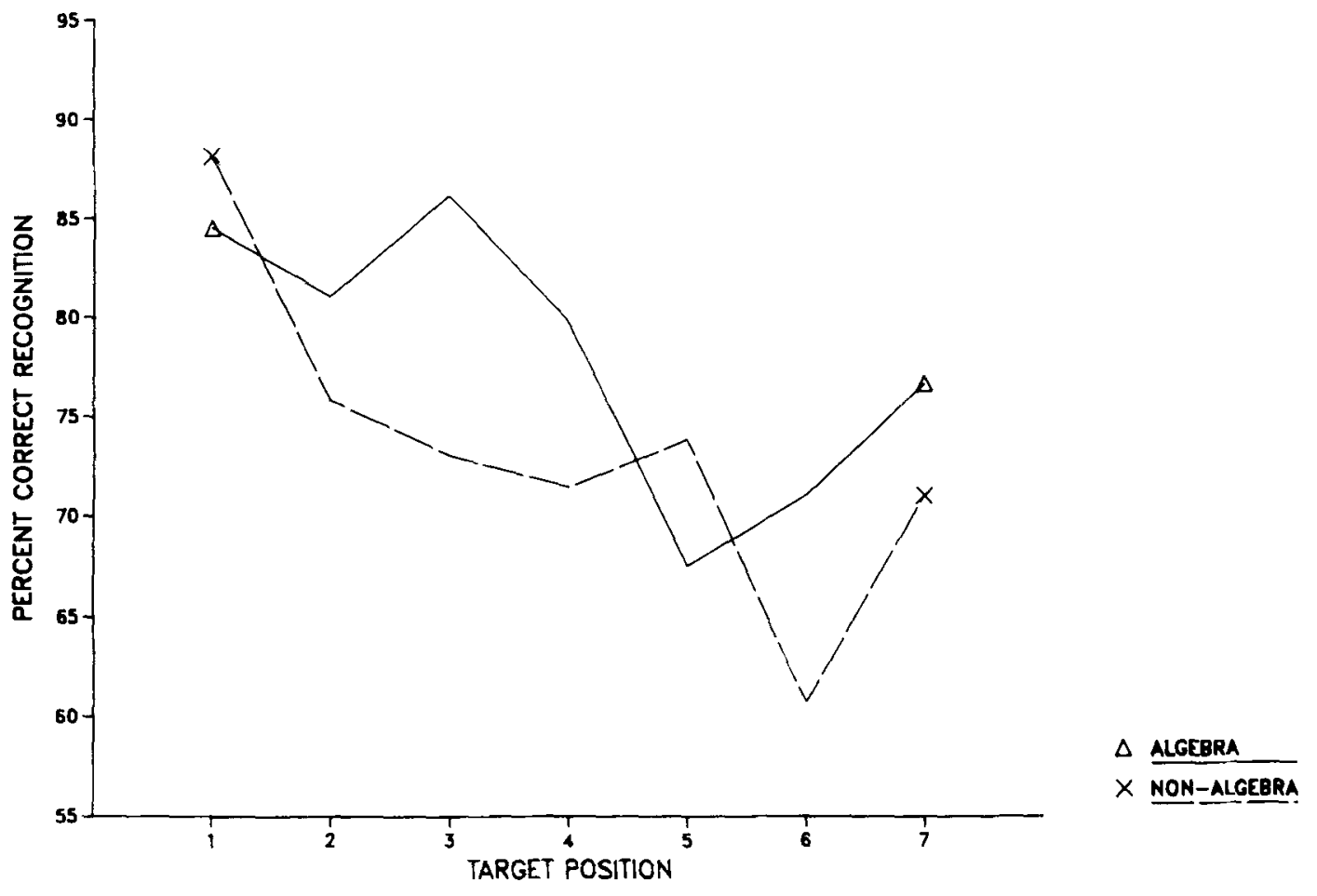

Figure 4. The percentage of correct recognition as a function of stimulus type and serial target position.

$\left(C I_{.95}=\left[-0.009<\mu_{\mathrm{A}}-\mu_{\mathrm{NA}}<0.030\right]\right)$. However, a multiple contrast analysis shows that the context of algebra yielded superior performance across both of the intercategorically tested different and categorical trials. [The intercategorical stimulus-type effects were significantly larger than the intracategorical effect: $F(1,24)=5.81$, $M S \mathrm{e}=.299, p<.025]$.

Across all three of the trial types, it was found that algebraic characters were recognized more accurately than were characters in nonalgebraic strings $(78.1 \%$ vs. $73.4 \%)$. This effect was highly significant $[F(1,13)=$ $43.44, M S e=.132, p<.0001]$, as was the main effect for serial position $[F(6,78)=5.08, M S e=1.292$, $p<.0005]$. As for the other two factors, the effect of target category was nonsignificant $(76.6 \%$ correct for numerals, $74.8 \%$ for letters), and the effect due to trial type was of marginal significance $[F(2,26)=3.19, M S \mathrm{~S}$ $=.769, p=.058]$. Of the remaining effects, a stimulus type $\times$ position interaction was evidenced $[F(6,78)=$ $5.14, M S e=.376, p<.0005$; see Figure 4], as well as a trial type $\times$ position interaction $[F(12,156)=3.41$, $M S e=.506, p<.0005 ;$ see Figure 5]. Although they may merely reflect ceiling effects, these results indicate that target position had a greater effect on both categorical trials and those involving nonalgebraic stimuli.

Mathematical modeling. As mentioned earlier, a syntactic contextual process could be relatively independent of bottom-up character identification processes. This attractively simple possibility was tested via the following task analysis and modeling effort.
Accurate performance on a given trial of this experiment was thought to be mediated by up to three forms of information. (1) For all trial types, a correct answer would result if the subject could explicitly recall the character originally presented in the probed position. (2) On trials that employed algebraic stimuli and intercategorical alternatives, the subject could utilize the syntactic context of algebra in order to respond accurately. (3) For trials that employed explicit characters as alternatives, differential feature knowledge (i.e., partial, yet discriminative) would permit the subject to answer correctly. Figure 6 depicts these sources of information in the form of a binary decision tree. Its branches are labeled in terms of the following stochastic parameters: $R$ is the probability of explicitly recalling the probed character, $C$ is the probability of effectively utilizing an algebraic stimulus's syntactic context, and $F$ is the probability of a correct response based solely upon a discriminative comparison of the features of two specific alternatives.

If the hypothesis of an independent contextual process is correct, then feature information must function in the same way for both algebraic and nonalgebraic stimuli. In other words, the $F$ parameter must be unitary. However, if an algebraic feature parameter $\left(F^{\prime}\right)$ could be found to be statistically distinct from a nonalgebraic feature parameter $\left(F^{\prime \prime}\right)$, the independence hypothesis would be disconfirmed. These considerations led to the adoption of the following mathematical function as a general stochastic model $(R C F F)$ of the performance reported above: 


$$
\begin{aligned}
P & =R+C(1-R)+F^{\prime}(1-R)(1-C) \\
& +F^{\prime \prime}(1-R)(1-C)\left(1-F^{\prime}\right) \\
& +.5(1-R)(1-C)\left(1-F^{\prime}\right)\left(1-F^{\prime \prime}\right)
\end{aligned}
$$

Because different variables within this formula for the proportion of correct responding $(P)$ should be set to zero for specific contexts and trial conditions, ${ }^{7}$ six new formulas result:

$$
\begin{aligned}
& \text { Categ/Nonalg: } \\
& P 1=R \\
& +.5(1-R) \\
& \text { Categ/Alg: } \\
& P 2=R+C(1-R) \quad+.5(1-R)(1-C) \\
& \text { Same/Nonalg: } \\
& P 3=R \quad+F^{m}(1-R) \quad+.5(1-R)\left(1-F^{\prime \prime}\right) \\
& \text { Same/Alg: } \\
& P 4=R \quad+F(1-R)+.5(1-R)\left(1-F^{\prime}\right) \\
& \text { Diff/Nonalg: } \\
& P 5=R \quad+F^{m}(1-R) \quad+.5(1-R)\left(1-F^{\prime \prime}\right)
\end{aligned}
$$

The general model was applied by using a computer program that searches a space of parameter values in order to minimize the function $-2\left[\ln \left(L_{i}\right)\right]$, where $L_{i}$ is the variably constrained likelihood function
$L_{1}=P 1^{s 1}(1-P 1)^{F 1} \times P 2^{s 2}(1-P 2)^{F 2} \times \ldots \ldots \ldots \times P 6^{s 6}(1-P 6)^{\text {st }}$

and where $S_{n}$ and $F_{n}$ correspond to the frequency of successes and failures for a particular sort of condition/stimulus conjunction. The stimulus type $\times$ trial type data from Experiment 2 provide values for $S 1$ through $S 6, F 1$ through $F 6$, and estimates for $P 1$ through $P 6$ (from Figure $3 ; .701, .776, .741, .752, .760$, and .815 , respectively). These values were used to calculate an atheoretical estimate (3886.74) of the function $-2\left[\ln \left(L_{0}\right)\right]$.

For two likelihood values, $L^{\prime}$ and $L^{\prime \prime}$, where $L^{\prime \prime}$ results from restricting the model that yields $L^{\prime}$, $-2\left[\ln \left(L^{\prime \prime}\right)-\ln \left(L^{\prime}\right)\right]$ is distributed asymptotically as $\chi^{2}$. Thus, stepwise statistical comparisons were made between the value of $-2\left[\ln \left(L_{0}\right)\right]$ and the corresponding values of minimized functions that are based upon specific theoretical models. In particular, it was found that the full fourparameter model ( $R C F F$ ) accounted for the subjects' data quite well $\left[x^{2}(2)=.55, p>.75\right]$. More interesting, though, is the fact that the constraint $F^{\prime \prime}=F^{\prime \prime}$ (model $R C F$ ) does not increase the lack of fit $\left[\chi^{2}(3)=.55, p>.90\right]$. This finding indicates that $F^{\prime}$ and $F^{\prime \prime}$ are not statistically distinct, and that the context of algebra does not significantly interact with feature-based character recognition.

It is also of interest to determine which of the basic parameters are needed to adequately model the obtained data. Setting both $F^{\prime}$ and $F^{\prime \prime}$ equal to zero (model $R C$ )

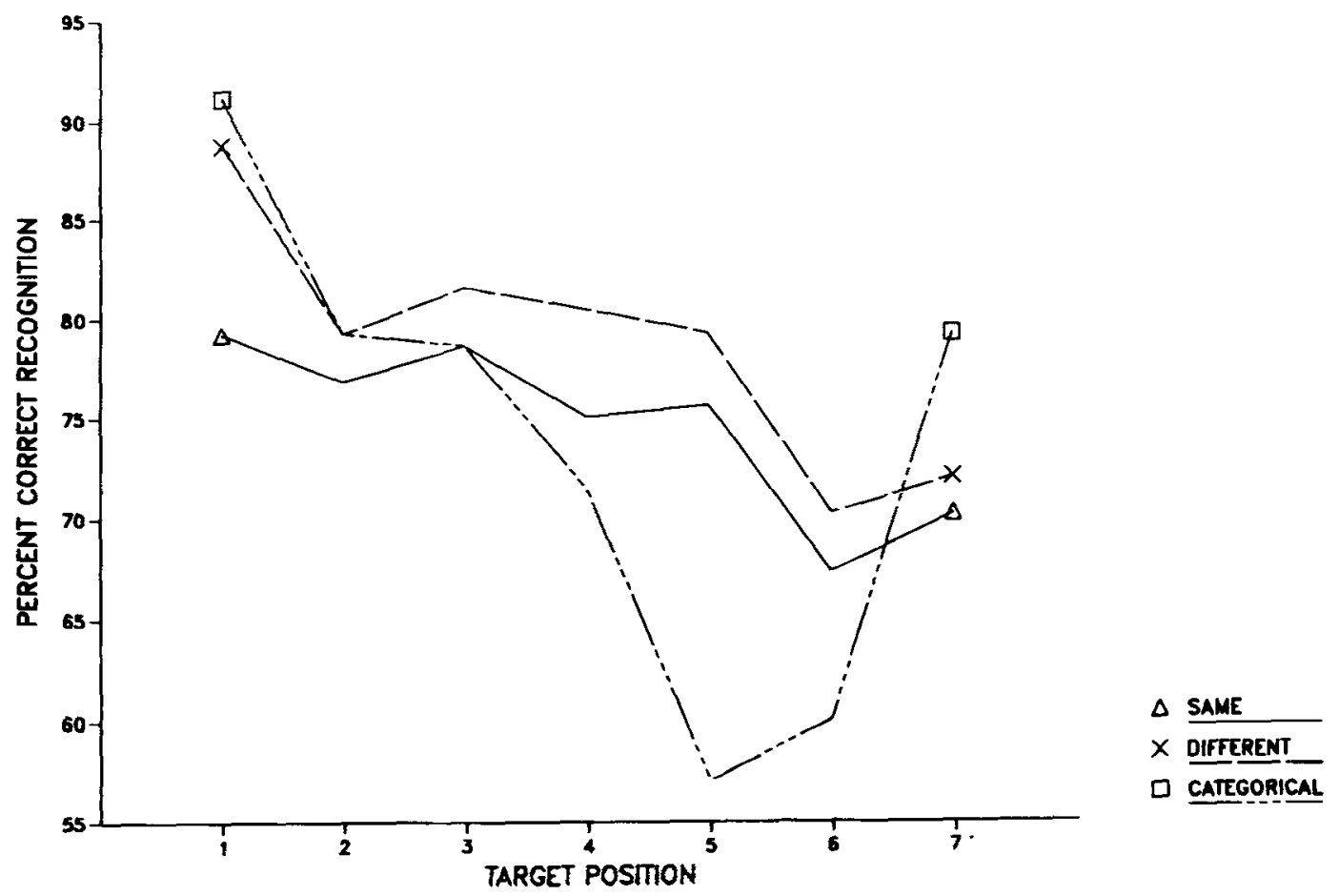

Figure 5. The percentage of correct recognition as a function of trial type and serial target position. 


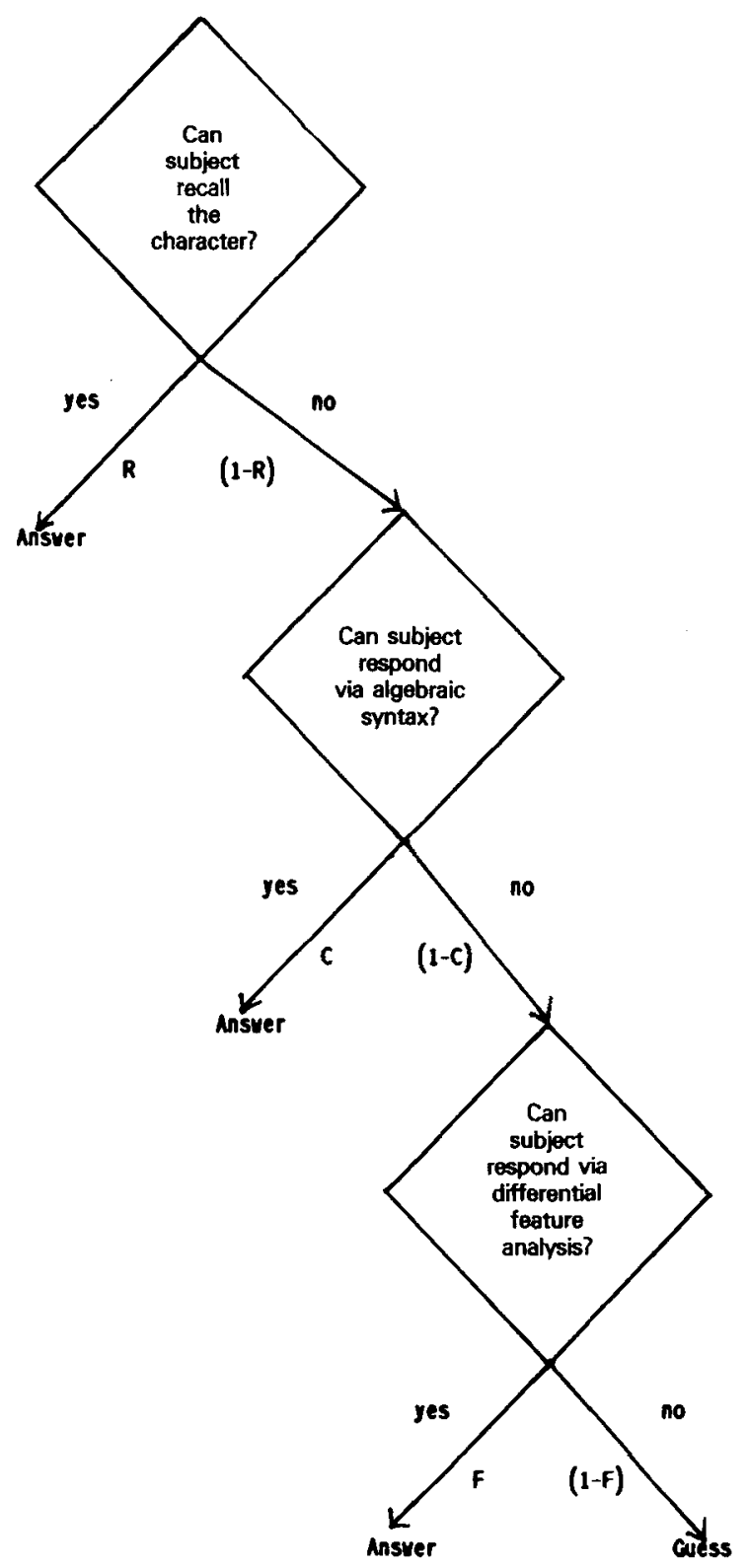

Figure 6. A binary decision tree indicating the utility of the three forms of information (see text for parametric descriptions) thought to mediate the performance exhibited during Experiment 2.

brought about a significant jump in the minimized function's value, and so exhibited a lack of fit for this model $\left(\chi^{2}(1)=8.47, p<.01\right.$, compared to the $R C F$ model; $\chi^{2}(4)=9.02, .10>p>.05$, compared to $\left.-2\left[\ln \left(L_{0}\right)\right]\right)$. Since constraining $F^{\prime}$ to $F^{\prime \prime}$ and $C$ to zero (model $R F$ ) also increased the lack of fit $\left[X^{2}(4)=19.40, p<.001\right]$, the $R C F$ model

$P=R+C(1-R)+F(1-R)(1-C)+.5(1-K)(1-F)(1-C)$

is clearly the best fitting and most parsimonious (yielding estimates of $.700, .776, .751, .751, .751$, and .814 , respectively, for $P 1$ through $P 6$ ). Table 3 presents the likelihood values and parameter estimates obtained for each of the above models.

Individual differences were also examined, using the statistical model. Although the sum of the subjects' unrestricted likelihood functions was not ill-fit by the group's unrestricted function $\left(X^{2}(78)=76.32, p>.50 ;-2\left[\ln \left(L^{\prime}\right)\right]\right.$ $=3810.42$ and $\left.-2\left[\ln \left(L^{\prime \prime}\right)\right]=3886.74\right)$, in modeling the individuals, it became apparent that not all of them used the postulated information sources to the same degree. ${ }^{8}$ From the perspective of the $R C F$ model, intersubject homogeneity was somewhat low $\left[x^{2}(39)=49.98\right.$, $.10>p>.05]$. Upon closer analysis, it was found that, although 8 of the subjects' data were best fit by the $R C F$ model, 5 were fit equally well with the $F$ parameter set to zero (via the $R C$ model), and one $R F$ subject's data were adequately fit with the $C$ parameter set to zero. In accordance with this analysis, and relative to $R C F$ subjects, the performance of the $R C$ subjects was less accurate on those (same and different) trials in which feature-based discrimination would have been of assistance than on (categorical) trials for which such a process was not appropriate. Figure 7 depicts this configuration of data.

\section{Discussion}

The lack of an algebraic effect for Experiment 2's same condition convincingly replicated the comparable result from Experiment 1: There is no evidence to support the existence of a strict algebraic analogue of the wordsuperiority phenomenon. This absence of effect is especially remarkable, given that the context of algebra yielded significantly heightened performance across both the different and the categorical trials. Without these data, one might have argued that Experiment 1's negative finding merely reflected a strategic deficiency, and that contextually based responding during the same trials was possible, but that such processes were of relatively low cognitive utility.

Superficially, the lack of an algebra-superiority effect signifies that the perception of a specific character is not enhanced by embedding it in an algebraic context. With respect to the processing of algebraic expressions, however, the lack of effect has somewhat broader implications. It suggests that, during brief stimulus exposures, there exists no top-down interaction between information about the syntactic nature of algebraic expressions and the detection of an individual character's visual features. These data contradict the hypotheses derived from models that include such interactive processes.

Clearly, no model incorporating a facile fixed lexicon could adequately account for the findings. Similarly, the

Table 3

Likelihood Values and Parameter Estimates Obtained for the Various Stochestic Modets Examined

\begin{tabular}{lccccccc}
\hline & & \multicolumn{5}{c}{ Parameter } \\
\cline { 3 - 7 } Model & $-2\left[\ln \left(L_{i}\right)\right]$ & $r$ & $C$ & $F$ & $F^{*}$ & $F^{*}$ \\
\hline$R C F$ & 3887.29 & .400 & .253 & .170 & & \\
$R C F F$ & 3887.29 & .401 & .252 & & .168 & .172 \\
$R C$ & 3895.76 & .477 & .216 & & & \\
$R F$ & 3906.14 & .476 & & .110 & & \\
\hline
\end{tabular}




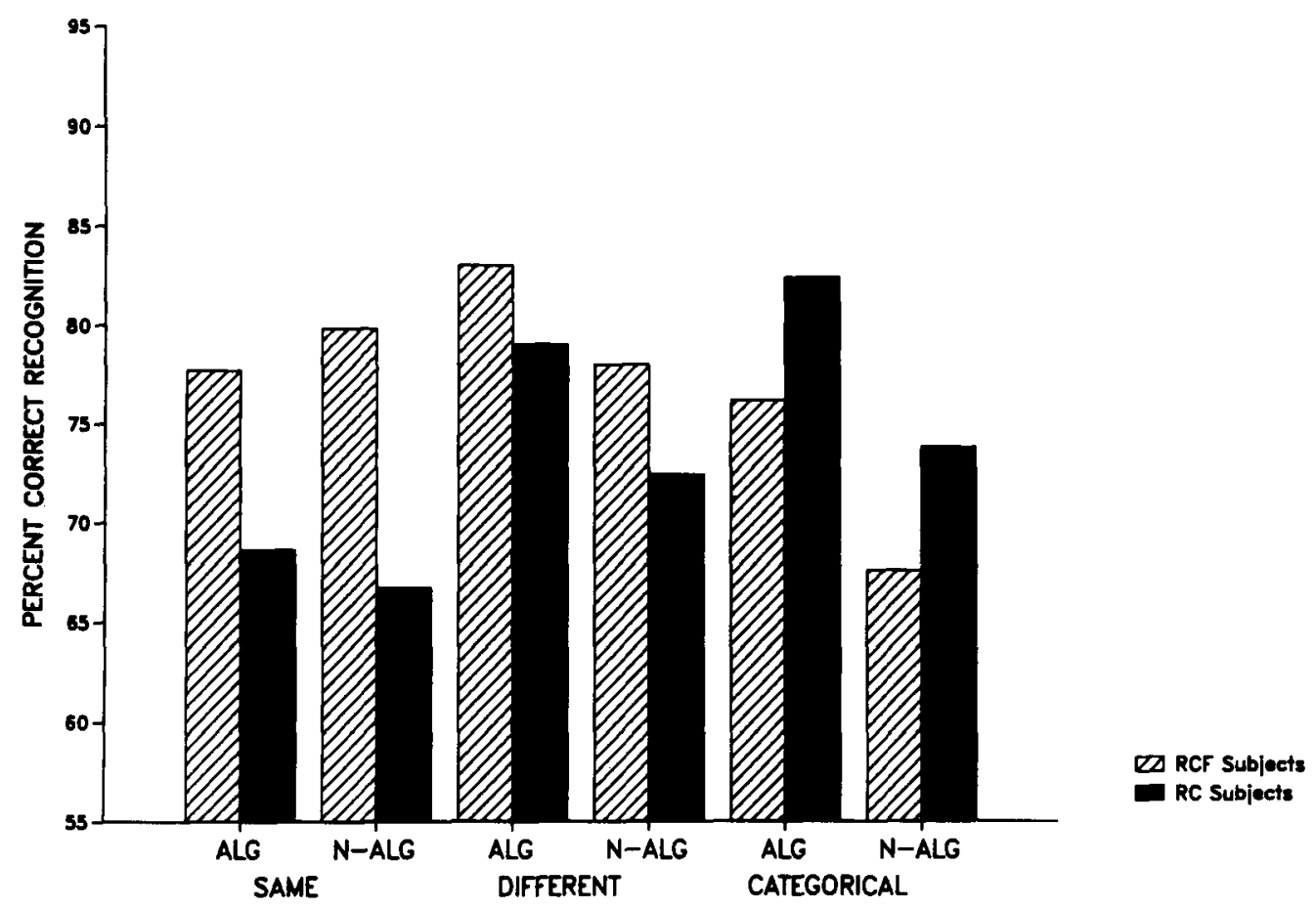

Figure 7. Subjects' recognition performance, as a function of stimulus type and trial type, as well as $R C F$ and $R C$ classification.

aforementioned interactive brand of generically structured lexicon also fails to account for the missing algebrasuperiority effect. Such a lexicon would embody a kind of orthography for algebra; therefore, one could have argued that knowing the category of a target character would serve to enhance its perception, because such knowledge would reduce its positional uncertainty and enhance the assignment of its features to the targeted position. ${ }^{9}$ This selective reduction of lateral interference would also yield a prediction of contextual enhancement for the algebraic strings of the same condition. The lack of such a phenomenon, however, strongly suggests that the structural context of algebra does not assist in the feature-based discrimination of two characters. ${ }^{10}$

With respect to the generative parse model, the results of Bernard's (1983) experiment must be reinterpreted. His recall data suggest that algebraic strings are better remembered due to generated representations and their associated chunking strategies. However, were such well-formed representations available after exposures of $120 \mathrm{msec}$, we would have again expected to find a contextual enhancement effect across the same trials. This was not the case; therefore, it seems that the generative parsing mechanisms evidenced in the recall procedure do not influence character recognition within the temporal parameters employed in this study. Such mechanisms would appear to be invoked later, perhaps only after each individual character has been adequately recognized.

One might assert that the structural context of algebra provides for no intercharacter discrimination at all, were it not for the algebraic effects found in the two new trial conditions in Experiment 2. (Recall that the main effect for stimulus type was actually due to their two subeffects.) These results indicate that subjects are able to utilize contextual cues when choosing between intercategorical alternatives. The conceptual surprise comes when one juxtaposes (as was done via the mathematical modeling) these results with the lack of an effect for the same condition. The conjunction indicates that subjects were occasionally capable of knowing a target's category without knowing its identity. Put another way, the categorical information embodied by the parameter $C$ was independent of the feature-based information embodied by the parameter $F$.

Finally, it may be worthwhile to note some divergent findings of minor theoretical interest. Although its effects were quite pronounced in the first experiment, in Experiment 2 target category played no statistical role, not even significantly interacting with the other variables. ${ }^{11} \mathrm{On}$ the other hand, beyond the standard main effect, the observed effects involving serial position are not easily interpreted. Although Ranney (1984) proposed several processes to account for these findings, the hypotheses are quite tenuous.

\section{A MODEL FOR THE INITIAL PERCEPTION OF ALGEBRAIC EXPRESSIONS}

In this section I present an informal processing model that accounts for the present study's basic findings. Although mildly speculative, the model is primarily offered (with respect to the mathematical modeling) as a complementary interpretation of the available data. 
From the previous predictions and the configuration of the obtained results, it is apparent that parsers of algebra are sensitive to the categorical/syntactic structure of algebraic expressions. Still, one wonders how a subject can bring such knowledge to bear upon a given stimulus. It seems plausible to suggest that an expression's operators provide the loci from which contextual enhancement mechanisms may be invoked. Responses from the subjects' retrospective interviews were consistent with this hypothesis.

A number of the students mentioned that they tried to "ignore" a stimulus's operating symbols and intentionally focus on a string's numerals and letters-the "meaningful" or "content" characters. If this was indeed a prevalent perceptual strategy, a subject would effectively be segmenting the stimulus into units composed of those characters. For algebraic expressions, the resulting units would always be regular algebraic terms, whereas for the nonalgebraic strings, such segments would be acceptable terms much less frequently.

The terms employed in this study could consist only of five basic forms, and encompassed a maximum of three horizontal positions; single-character terms were either letters or numerals, double-character terms were either numeral-letter or letter-letter pairs, and triple-character terms were always numeral-letter-letter sequences. Were an algebraic expression to be parsed into such terms, a segment's length alone would be a good indicator of its categorical constituents: the first character in a term was usually a numeral, the rest always letters. Thus, a kind of local syntax could drive the knowledge of a particular character's categorical denomination. The syntax would, presumably, have been acquired over the course of a subject's past experience with algebraic expressions, and therefore would be of considerably less use (or even a hindrance) in the recognition of characters embedded in nonalgebraic strings. This local syntax is one whose intercharacter contingencies are even more predictable than those of words.

On the basis of this version of the function of algebraic syntax, I propose a processing model to account for the results of the present study. Its design is constrained by the results of the mathematical modeling described earlier. Figure 8 graphically depicts the basic flow of information in the proposed system. It should be noted that the model's critical feature is a contextual process that is generally independent of bottom-up recognition processes (e.g., complete character recognition). The process merely requires information about the location of a stimulus's operational symbols. Upon receipt of this information, it sets up categorical expectancies for the characters that compose the

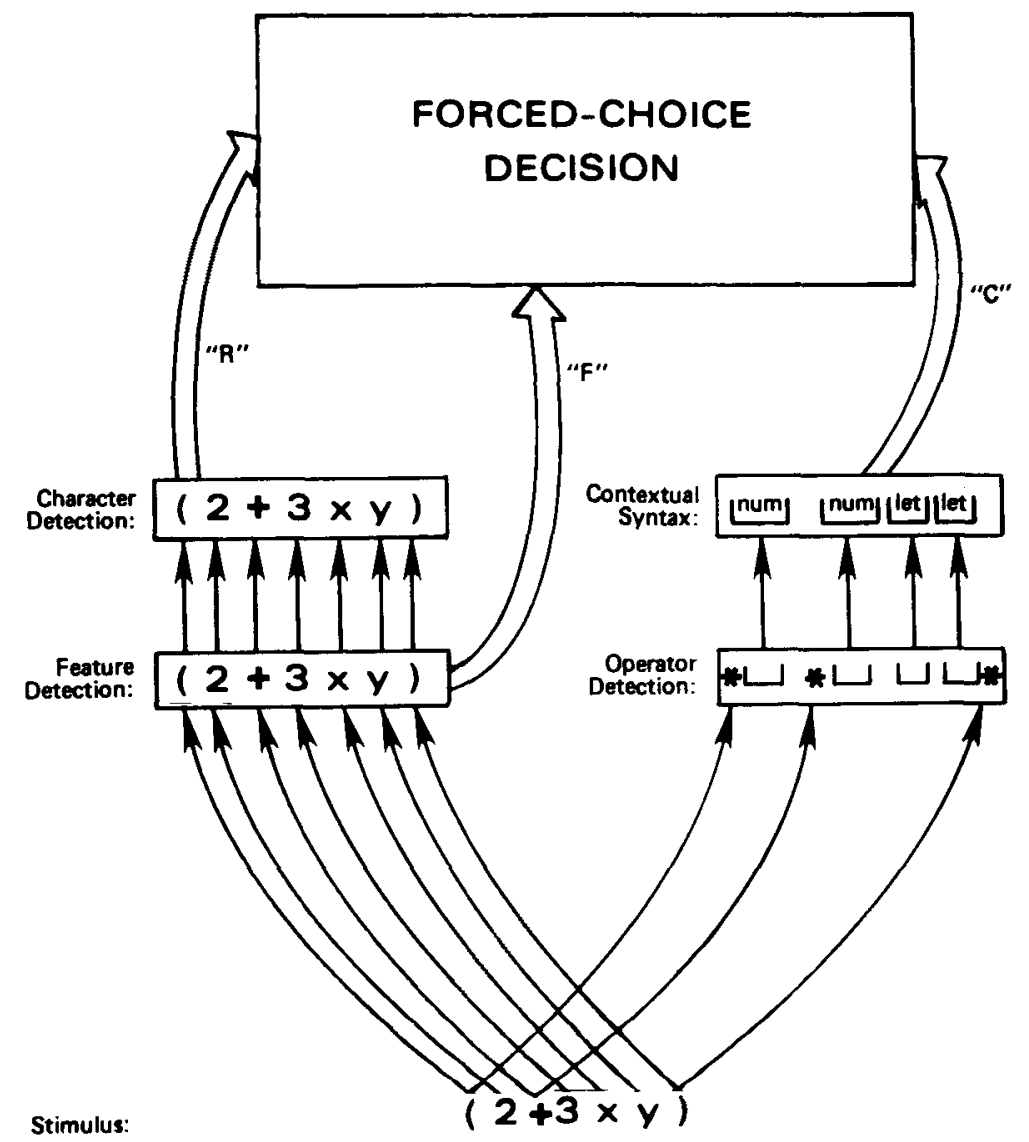

Figure 8. A schematic depiction of the proposed processing model during a rudimentary parsing of the expression $(2+3 x y)$. 
resulting segments. I propose that these expectancies are the embodiment of the syntactic-contextual parameter $C$ from the mathematical modeling. The model's featuredetection and character-detection processes provide output that corresponds to the aforementioned $F$ and $R$ parameters, and are comparable to McClelland and Rumelhart's (1981) feature- and letter-detection processes.

In a number of respects, this model is similar to the sophisticated guessing theories (Rumelhart \& Siple, 1974; Thompson \& Massaro, 1973; Wheeler, 1970) that have been proposed (and apparently rejected; see Johnston, 1978, and Adams, 1979) to account for the wordsuperiority effect. It was thought that the effect might be the result of guesses based on the combination of a letter's features and the orthographic constraints of its surrounding word context. In contrast to guessing on the basis of lexical orthography, however, the model of algebraic perception proposed here hypothesizes a local form of syntax that independently supplements feature-based perceptual processes.

The model also entails the inherent assumption that operational characters such as ( and + are detected more quickly than are "content" characters such as 5 and $Y$. Yet these characters need not be completely identified; perhaps they are merely categorized and located. This conjecture is not without some empirical support. Jonides and Gleitman (1976) found that, during between-category search (letters vs. digits), subjects appear to rapidly "tag" the positions of characters from the targeted category well before the characters are fully identified. For brief durations, then, we might view operational characters as a form of punctuation, serving the same perceptual function in algebra as do spaces, hyphens, and slashes in the parsing of words in English sentences. (Note that Fisher, 1975, found that the reading of prose is slowed by a factor of three when the interword spaces are removed.) Furthermore, most of these symbols comprise relatively few features, which could also result in speeded detection. But even if the operational characters were more complex than those to be segmented, data from Jonides and Gleitman (1972) and others seem to suggest that their existence as a class of symbols may be enough to denote them as "ground" to the "figures" of terms.

Certainly, the model proposed is in ned of further empirical support, and may eventually be disconfirmed. With respect to the data available, though, it provides a coherent account of the function of the structural context of algebra during the initial stages of algebraic parsing.

\section{REFERENCES}

ADAms, M. J. (1979). Models of word recognition. Cognitive Psychol ogy, 11, 133-176.

BERNARD, J. (1983). An essay on perception and understanding of mathematical symbolism. Intemational Joumal for Mathematics Education in Science \& Technology, 14, 489-496.

CAtTell, J. M. (1885). The inertia of the eye and brain. Brain, 8, 295-312.
Chase, W. G., \&imon, H. A. (1973). Perception in chess. Cognitive Psychology, 4, 55-81.

DunCAN, J. (1983). Category effects in visual search: A failure to replicate the "oh-zero" phenomenon. Perception \& Psychophysics, 34, 221-232.

EGAN, D. E., SCHWARTZ, B. I. (1979). Chunking in recall of symbolic drawings. Memory \& Cognition, 7, 149-158.

EsTes, W. K. (1975). Memory, perception, and decision in letter identification. In R. L. Solso (Ed.), Information processing and cognition: The Loyola symposium (pp. 3-30). Hillsdale, NJ: Erlbaum.

Estes, W. K., AllmeYer, D. H., \& Reder, S. M. (1976). Serial position functions for letter identification at brief and extended exposure durations. Perception \& Psychophysics, 19, 1-15.

FISHER, D. F. (1975). Reading and visual search. Memory \& Cognition, 3, 188-196.

INGLNG, N. W. (1972). Categorization: A mechanism for rapid information processing. Joumal of Experimental Psychology, 94, 239-243.

Johnston, J. C. (1978). A test of the sophisticated guessing theory of word perception. Cognitive Psychology, 10, 123-153.

Jonides, J., Glemtman, H. (1972). A conceptual category effect in visual search: $\mathrm{O}$ as letter or as digit. Perception \& Psychophysics, 12 , 457-460.

Jonides, J., \& Gleitman, H. (1976). The benefit of categorization in visual search: Target location without identification. Perception \& Psychophysics, 20, 289-298.

KrUEGER, L. E. (1984). The category effect in visual search depends upon physical rather than conceptual differences. Perception \& Psychophysics, 35, 558-564.

Massaro, D. W. (1979). Letter information and orthographic context in word perception. Joumal of Experimental Psychology: Human Perception \& Performance, 5, 595-609.

McClelland, J. L., \& Rumelhart, D. E. (1981). An interactive activation model of context effects in letter perception: Part 1. An account of a basic findings. Psychological Review, 88, 375-407.

MilleR, G. A., ISARD, S. (1963). Some perceptual consequences of linguistic rules. Joumal of Verbal Leaming \& Verbal Behavior, 2, 217-228.

PaAp, K. R., Newsome, S. L., McDonald, J. E., Schyaneveldt, R. W. (1982). An activation-verification model for letter and word recognition: The word-superiority effect. Psychological Review, 89, 573-594.

RANNEY, M. (1984). Structural context in perception: Syntax in the recognition of graphemic strings. Unpublished master's thesis, University of Pittsburgh.

Reicher, G. M. (1969). Perceptual recognition as a function of meaningfulness of stimulus material. Joumal of Experimental Psychology, 81 , 275-280.

Rertman, J. S. (1976). Skilled perception in Go: Deducing memory structures from inter-response times. Cognitive Psychology, 8, 336-356.

Rumelhart, D. E., MCClellaNd, J. L. (1982). An interactive activation model of context effects in letter perception: Part 2 . The contextual enhancement effect and some tests and extensions of the model. Psychological Review, 89, 60-94.

Rumelhart, D. E., \& SiPLE, P. (1974). The process of recognizing tachistoscopically presented words. Psychological Review, 81, 99-118.

Thompson, M. C., Massaro, D. W. (1973). Visual information and redundancy in reading. Joumal of Experimental Psychology, 98, 49-54.

WheEleR, D. D. (1970). Processes in word recognition. Cognitive Psychology, 1, 59-85.

WHITE, M. J. (1977). Identification and categorization in visual search. Memory \& Cognition, 5, 648-657.

\section{NOTES}

1. The stimuli included no character-size spaces, as one might find in an algebra textbook.

2. Subjects typically performed such that the stimuli were eventually viewed for less than $110 \mathrm{msec}$ each.

3. A more complete discussion of this experiment is provided in Ranney (1984). 
4. Another methodological point: As in Experiment 1, the present procedure probed only those stimulus positions that had contained either a letter or a numeral. Although testing for positions that had contained operational characters (parentheses and operators) was considered, the notion was rejected for the following reason: Many studies have shown that subjects make clear categorical distinctions between letters and numerals (Duncan, 1983; Jonides \& Gleitman, 1972; White, 1977), and that the perception of such characters can be enhanced on the basis of their categorical membership. The most basic and robust finding (e.g., Ingling, 1972) is that a target letter is more rapidly identified when embedded in a field of numbers (as in a numeral amongst letters) than when embedded in a field of its own conceptual-taxonomic category. There was little reason to believe that subjects include operational characters such as + and (in the same conceptual category; therefore, employing such characters as either targets or foils, particularly in the different and categorical conditions, might require the subject to adopt an unnatural taxonomy. If this were true, probing positions that held operational characters could introduce aberrant perceptual and strategic processes into an already complex task.

5. Formulas $2 a$ through $2 f$ and their associated discussion further elucidate these characteristics.
6. The present experiment's exposure durations were quite comparable to those of Experiment 1. Most subjects were eventually able to maintain the performance criterion while viewing the stimuli for less than $120 \mathrm{msec}$.

7. The $C$ parameter was set to zero for nonalgebraic and same trials; $F$ was set to zero for nonalgebraic and categorical trials; $F^{*}$ was set to zero for algebraic and categorical trials.

8. Of course, the differential use of available knowledge need not be viewed as a basic processing difference. The individual differences observed might well be the result of differences in salience among the various sources of information.

9. This type of mechanism is similar to that proposed by Estes, Allmeyer, and Reder (1976) for word recognition.

10. Massaro (1979) provides analogous results with respect to word recognition, in that the information provided by feature analysis was found to be independent of that provided by lexical context.

11. Krueger (1984) discussed the capriciousness of results regarding the relative ease of processing letters and digits.

(Manuscript received January 21, 1986; revision accepted for publication June 20,1986 .) 